\title{
Liquid biopsy in tumors: opportunities and challenges
}

\author{
Yi Bai, Haitao Zhao \\ Department of Liver Surgery, Peking Union Medical College Hospital, Chinese Academy of Medical Sciences and Peking Union Medical College, \\ Beijing 100730, China \\ Correspondence to: Haitao Zhao, MD. Department of Liver Surgery, Peking Union Medical College Hospital, Chinese Academy of Medical Sciences \\ and Peking Union Medical College (CAMS \& PUMC), 1 Shuaifuyuan, Wangfujing, Beijing 100730, China. Email: ZhaoHT@pumch.cn. \\ Comment on: Gandara DR, Paul SM, Kowanetz M, et al. Blood-based tumor mutational burden as a predictor of clinical benefit in non-small-cell \\ lung cancer patients treated with atezolizumab. Nat Med 2018;24:1441-8.
}

Submitted Oct 21, 2018. Accepted for publication Nov 12, 2018.

doi: 10.21037/atm.2018.11.31

View this article at: http://dx.doi.org/10.21037/atm.2018.11.31

The emergence of non-invasive diagnostic techniques for tumors (1), such as liquid biopsy, marks a major step forward for humans on the road to conquering tumors. From the 2016 Food and Drug Administration (FDA) approval of the first liquid biopsy products based on EGFR gene mutations (2), by August this year, tumor mutation burden in blood (bTMB) was confirmed to predict the effectiveness of immunotherapy, and liquid biopsy has already made a big splash in the field of cancer treatment (3). Liquid biopsy has the advantages of non-invasive, rapid, precise, and especially real-time. It can also overcome the heterogeneity of tumors and may replace tissue biopsy in the future. Liquid biopsy currently mainly includes circulating tumor cells (CTC), circulating tumor DNA (ctDNA) and exosomes, and samples can be derived from blood, urine, saliva and the like. Liquid biopsy has broad application prospects in the diagnosis and treatment of cancer, including early diagnosis screening, tumor heterogeneity, drug resistance, and establishment of targets (4).

The mutations and amplifications caused by ctDNA are closely related to the occurrence and development of tumors and can be used to monitor the therapeutic effect $(5,6)$. However, in targeted therapy, the detection rate of mutations is relatively low. In addition, the circulating DNA molecules in the blood are usually much lower than the non-cancer-related DNA fragments in the blood, which makes it very difficult to detect them, especially in the early stages of cancer. Previous studies have found that accurate tumor information can only be obtained when the abundance of ctDNA in cell-free DNA (cfDNA) is greater than or equal to $10 \%$. However, except for some advanced tumors that release large amounts of ctDNA, the abundance of ctDNA in most tumor patients does not meet this criterion $(4,7,8)$. A team led by University of Cambridge scientist Nitzan Rosenfeld enriches plasma-specific fragment length cfDNA in vitro and then sequenced it. It was found that sequencing results were able to distinguish tumor blood samples from healthy blood ones, the area under the curve exceeded 0.99. Enriched normal cfDNA assays are less than 0.8 . In addition, this enrichment method allows liquid biopsy to detect cancer at an earlier stage (9).

Recently, Gandara et al. (3) first confirmed that the tumor mutation load in plasma can be accurately and repeatedly measured and correlated with the efficacy of immunological checkpoint inhibitors. The effectiveness of bTMB in predicting the efficacy of immunotherapeutic drugs was confirmed. For the prediction of the efficacy of immunotherapy, it provides a less invasive, more reproducible alternative detection program. This means that cancer patients receiving immunotherapy will be expected to have a more convenient and accurate method for predicting the efficacy in the near future. In 2016, Bristol-Myers Squibb (BMS) announced that its PD-1 antibody, Opdivo, did not reach the primary clinical endpoint in the phase III clinical trial of non-small cell lung cancer (10). In this study, the researchers found that for patients with advanced lung cancer with PD-L1 $\geq 5 \%$ and untreated, Opdivo failed to prolong progressionfree survival compared with standard chemotherapy. Of note, they reanalyzed the trial data and found that for those patients with high TMB levels, the results of tumor remission and survival benefit were significantly better than 
those of chemotherapy after treatment with Opdivo. The reason why immunotherapy can exert good anticancer effect depends mainly on the recognition of cancer cell-specific antigen by immune cells. Hence, in theory, for those cancer patients with more mutations, the more neo-antigens the cancer produces, and the more likely it is to be recognized by immune cells $(11,12)$. In other words, the higher the $\mathrm{TMB}$, the more patients may benefit from immunotherapy. According to these, Jaffee et al. concluded that there was a significant positive correlation between $\mathrm{TMB}$ and response rate of immunological checkpoint inhibitor treatment after analyzing multiple clinical study data (13).

In this study, the authors developed a technical solution for detecting TMB in the blood and conducted a retrospective analysis of two large randomized trials as test (NCT01903993) and validation (NCT02008227) studies. Having compared bTMB of the two clinical cohorts with tissue-based TMB (tTMB), they confirmed that $\mathrm{tTMB}$ and bTMB were indeed positively correlated as a whole. After calculation, the researchers finally selected bTMB $\geq 16 \mathrm{mut} / \mathrm{Mb}$ as the threshold of the training set and continued to verify in the verification set. In the population with a validation set of $\mathrm{bTMB} \geq 16 \mathrm{mut} / \mathrm{Mb}$, the median overall survival of patients receiving atezolizumab immunotherapy was 13.5 months, while the median overall survival of patients receiving docetaxel was 6.8 months, which indicated that bTMB does indeed serve as a potential non-invasive biomarker for atezolizumab immunotherapy.

However, due to the rapid speed of clinical promotion of this method, related studies on the clinical effectiveness of the method have not been confirmed. Early in this year, the American Society of Clinical Oncology (ASCO) and the American College of Pathologists (CAP) organized a committee of experts to conduct a review of the published 1,338 clinical ctDNA tests. After evaluating the ctDNA detection method from three aspects including analytical validity, clinical validity, and clinical utility, they concluded that the current clinical efficacy of liquid biopsy techniques is very limited (14).

To sum up, liquid biopsy has become the focus of future research on precision diagnosis and treatment of tumors (15). However, there are still many problems to be solved at this stage. ctDNA detection can be used as a means of targeting drug treatment for drug resistance, providing more possibilities for the future. Liquid biopsy ctDNA methylation is very promising in the field of precise treatment of tumors (16). But as with any other test method, liquid biopsies also require large-scale cohort studies to determine their effectiveness and sensitivity to different diseases. Behind radical clinical applications, solid clinical effectiveness and clinical utility evaluations need to be kept up to date.

\section{Acknowledgements}

Funding: This work was supported by CAMS Innovation Fund for Medical Science (CIFMS) (2017-I2M-4-003), International Science and Technology Cooperation Projects (2015DFA30650 and 2016YFE0107100), Capital Special Research Project for Health Development (2014-2-4012), Beijing Natural Science Foundation (L172055), National Ten-thousand Talent Program and Beijing Science and Technology Cooperation Special Award Subsidy Project.

\section{Footnote}

Conflicts of Interest: The authors have no conflicts of interest to declare.

\section{References}

1. Li C, Li R, Zhang W. Progress in non-invasive detection of liver fibrosis. Cancer Biol Med 2018;15:124-36.

2. Odogwu L, Mathieu L, Goldberg KB, et al. FDA BenefitRisk Assessment of Osimertinib for the Treatment of Metastatic Non-Small Cell Lung Cancer Harboring Epidermal Growth Factor Receptor T790M Mutation. Oncologist 2018;23:353-9.

3. Gandara DR, Paul SM, Kowanetz M, et al. Bloodbased tumor mutational burden as a predictor of clinical benefit in non-small-cell lung cancer patients treated with atezolizumab. Nat Med 2018;24:1441-8.

4. Siravegna G, Marsoni S, Siena S, et al. Integrating liquid biopsies into the management of cancer. Nat Rev Clin Oncol 2017;14:531-48.

5. Jamal-Hanjani M, Wilson GA, McGranahan N, et al. Tracking the Evolution of Non-Small-Cell Lung Cancer. N Engl J Med 2017;376:2109-21.

6. Abbosh C, Birkbak NJ, Wilson GA, et al. Corrigendum: Phylogenetic ctDNA analysis depicts early-stage lung cancer evolution. Nature 2018;554:264.

7. Murtaza M, Dawson SJ, Tsui DW, et al. Non-invasive analysis of acquired resistance to cancer therapy by sequencing of plasma DNA. Nature 2013;497:108-12.

8. Bettegowda C, Sausen M, Leary RJ, et al. Detection of circulating tumor DNA in early- and late-stage human 
malignancies. Sci Transl Med 2014;6:224ra24.

9. Mouliere F, Chandrananda D, Piskorz AM, et al. Enhanced detection of circulating tumor DNA by fragment size analysis. Sci Transl Med 2018;10. doi: 10.1126/scitranslmed.aat4921.

10. Giroux Leprieur E, Dumenil C, Julie C, et al. Immunotherapy revolutionises non-small-cell lung cancer therapy: Results, perspectives and new challenges. Eur J Cancer 2017;78:16-23.

11. Fukui H. A new link between innate immunity and hepatic metabolism: attractive unknown place to visit. Hepatobiliary Surg Nutr 2017;6:264-7.

12. Carbone DP, Reck M, Paz-Ares L, et al. First-Line Nivolumab in Stage IV or Recurrent Non-Small-Cell

Cite this article as: Bai Y, Zhao H. Liquid biopsy in tumors: opportunities and challenges. Ann Transl Med 2018;6(Suppl 1):S89. doi: 10.21037/atm.2018.11.31
Lung Cancer. N Engl J Med 2017;376:2415-26.

13. Yarchoan M, Hopkins A, Jaffee EM. Tumor Mutational Burden and Response Rate to PD-1 Inhibition. N Engl J Med 2017;377:2500-1.

14. Merker JD, Oxnard GR, Compton C, et al. Circulating Tumor DNA Analysis in Patients With Cancer: American Society of Clinical Oncology and College of American Pathologists Joint Review. J Clin Oncol 2018;36:1631-41.

15. Ilmer M, Berger M. Avatars to personalized medicine: of mice and men. Hepatobiliary Surg Nutr 2017;6:347-9.

16. Sookoian S, Pirola CJ. Cell-free DNA methylation as liquid biopsy for the assessment of fibrosis in patients with nonalcoholic steatohepatitis: a gap between innovation and implementation. Hepatobiliary Surg Nutr 2017;6:117-21. 